\title{
Insights into Potential Mechanisms of Injury and Treatment Targets in COVID-19, SARS-Cov-2 Infection
}

Angelina Zhyvotovska\#, Denis Yusupov*, Robert Foronjy, Mohammed Nakeshbandi, Samy I McFarlane* and Moro Salifu

Department of Medicine, State University of New York (SUNY) Downstate Health Sciences University, Brooklyn, New York, USA**

\begin{abstract}
The severe acute respiratory syndrome coronavirus 2, SARS-CoV-2, is the most serious pandemic in modern times. The disease was first reported in January of 2020 in China's city of Wuhan, Hubei province, and since then it has spread worldwide. Given the rapid spread of the virus and the burden it has taken on the healthcare systems it has swept through, there is the need for a concise description of current understanding of the pathogenesis of organ failure in SARS-CoV-2 infection while acknowledging that more is yet to be uncovered. This review will not only inform decision making at the bedside but will also help illustrate potential therapeutic targets for research. We searched the available literature to-date, and present the pathophysiology underlying increased morbidity and mortality of SARS-CoV-2 infection in the lungs, heart and kidneys in a highly illustrated presentation that is easy-to-understand for the clinician, researcher, and student alike.
\end{abstract}

\section{Publication History:}

Received: May 13, 2020

Accepted: June 06, 2020

Published: June 08, 2020

\section{Keywords:}

COVID-19, Pathogenesis, SARSCoV-2, Acute Kidney Injury, Cardiac manifestation, Multiorgan failure

\section{Virology and Entry into a Host Cell}

To understand the origins of the COVID-19 pandemic we must first remember the severe acute respiratory syndrome coronavirus (SARS$\mathrm{CoV}$ ) which emerged in 2002. The two virus strains, SARS-CoV and SARS-CoV-2 are similar; phylogentic analysis has demonstrated that SARS-CoV-2 has approximately $76 \%$ nucleotide identity with SARS$\mathrm{CoV}$ [1]. Work by Hoffman et al. has shown that SARS-CoV-2 enters an identical spectrum of cell lines as SARS-CoV and its mechanism of entrance is similar. This suggests similarities in choice of entry receptors for both viruses. Both viruses depend on their spike (S) proteins for entry into a host cell. The S1 unit of the S protein attaches and binds to the host cell's receptor. Entry into the cell then requires priming of the $S$ protein by the host cell's cellular proteases which leads to a fusion of the viral and host cell's membranes leading to endocytic entry. The host cell receptor is angiotensin- converting enzyme 2 (ACE2) and the serine protease employed is TMPRSS2 [1] This entry causes cellular internalization of ACE2 and downregulation of these receptors: the importance of which will be described later. Normally, ACE2 is only scarcely present in the circulation in a soluble form; however, ACE2 is widely expressed and its receptors have been found in the arterial and venous endothelial cells and arterial smooth muscle cells of nearly every organ, including the lung, heart, kidney, and brain; and has been found in abundance in the oral and nasal mucosa, lung alveolar epithelial cells, enterocytes of the small intestines, cardiac myocytes, renal podocytes and cells of the proximal convoluted tubule [2-5]. This may, in part, help explain the array of systemic symptoms seen in COVID-19.

\section{Proposed Pathogenesis of SARS-CoV-2 in the Lung}

We propose that the virus likely first enters the host through the oral or nasal mucosa and then spreads either down to the gastrointestinal system or into the respiratory system before causing systemic infection in some patients. There is a wide spectrum of the severity of respiratory manifestations in patients infected with SARS-CoV-2; some patients may have a dry cough for a few days, while others suffer from acute respiratory distress syndrome (ARDS) requiring mechanical ventilation with high positive end expiratory pressures (PEEPs). The progression to ARDS may be spurred by the following mechanisms, illustrated in Figure 1. ACE2 is found in abundance on the alveolar epithelial cells, particularly in pneumocyte type II cells [5]. Though the exact mechanisms are unclear, based on the current literature, we hypothesize that cellular internalization and subsequent spread of the virus in the respiratory system leads to the following processes: 1) an increase in the ratio of levels of angiotensin converting enzyme 1 (ACE1) and angiotensin II compared to levels of ACE2 and angiotensin 1-7;2) a significant inflammatory response mediated by neutrophils, macrophages and CD8+ T cells leading to alveolar edema; 3 ) thrombus formation; 4) potential destruction of the pneumocyte type II cells [2,5-12].

Decreased ACE2 levels play a significant role in the progression to ARDS. ACE2 inactivates angiotensin II while generating angiotensin $1-7$, thus it downregulates the rennin-angiotensin system (RAAS) $[2,6]$. The subsequent, enhanced and unopposed, vasoconstriction may, in part, explain the atypical nature of the ARDS that develops in COVID-19. What we have witnessed in our institutions is similar to what has been observed by Gattinoni et al. in Italy; namely, that the lungs which develop ARDS retain a relatively high level of compliance. Despite reasonable lung compliance, severe hypoxemia persists even when using recruitment strategies such as high PEEP and prone positioning [13].

Dysregulated lung perfusion and hypoxic vasoconstriction via loss of ACE2 and the consequent increase of angiotensin II, the potent vasoconstrictor, to levels of angiotensin 1-7 can help explain some of the hypoxemia. Increased pulmonary inflammation and coagulation have been reported as effects of unopposed angiotensin II. In a study involving 12 patients, it was found that angiotensin II levels in the

"Corresponding Author: Prof. Samy I. McFarlane, College of Medicine, Department of Medicine, Division of Endocrinology, Internal Medicine Residency Program Director, State University of New York, Downstate Medical Center, 450 Clarkson Ave, Box 50, Brooklyn, New York, USA, Tel: 718-270-6707, Fax: 718-2704488; E-mail: smcfarlane@downstate.edu

\#Both authors equally contributed to this manuscript.

**Designated as the COVID-19 only hospital in New York City, NY during the pandemic.

Citation: Zhyvotovska A, Yusupov D, Foronjy R, Nakeshbandi M, McFarlane SI et al. (2020) Insights into Potential Mechanisms of Injury and Treatment Targets in COVID-19, SARS-Cov-2 Infection. Int J Clin Res Trials 5: 147. doi: https://doi. org/10.15344/2456-8007/2020/147

Copyright: (C) 2020 Zhyvotovska et al. This is an open-access article distributed under the terms of the Creative Commons Attribution License, which permits unrestricted use, distribution, and reproduction in any medium, provided the original author and source are credited. 
Citation: Zhyvotovska A, Yusupov D, Foronjy R, Nakeshbandi M, McFarlane SI, et al. (2020) Insights into Potential Mechanisms of Injury and Treatment Targets in COVID-19, SARS-Cov-2 Infection. Int J Clin Res Trials 5: 147. doi: https://doi.org/10.15344/2456-8007/2020/147

Page 2 of 9

plasma samples from SARS-CoV-2 infected patients was markedly elevated and linearly associated to viral load and lung injury [14]. Further aggravating this change in the ratio of Angiotnesin 1-7 to Angiotensin II, is that angiotensin II upregulates disintegrin and metalloproteinase 17 (ADAM17) which cleaves membraneanchored ACE2 [5]. This causes a loss of the catalytic activity of ACE2 on membranes. Elevated levels of circulating soluble ACE2 are a sign of increased activity of the RAAS system and are associated with a worse prognosis [5]. Additionally, loss of ACE2 can cause neutrophil accumulation which eventually contributes to ARDS and increased vascular permeability and thusly pulmonary edema [2]. This has clinical significance as it may inform future treatment strategies. For instance, studies in mice infected with avian influenza H5N1, showed that administration of recombinant human ACE2 decreased virus-induced lung injury [15]. Of additional interest, is that angiotensin II further interferes with adaptive immunity by activating macrophages, and other immune cells, causing increases of IL-6, TNF-alpha and other inflammatory cytokines [5].

\section{Proposed Pathogenesis of SARS-COV-2 causing Acute Respiratory Distress}

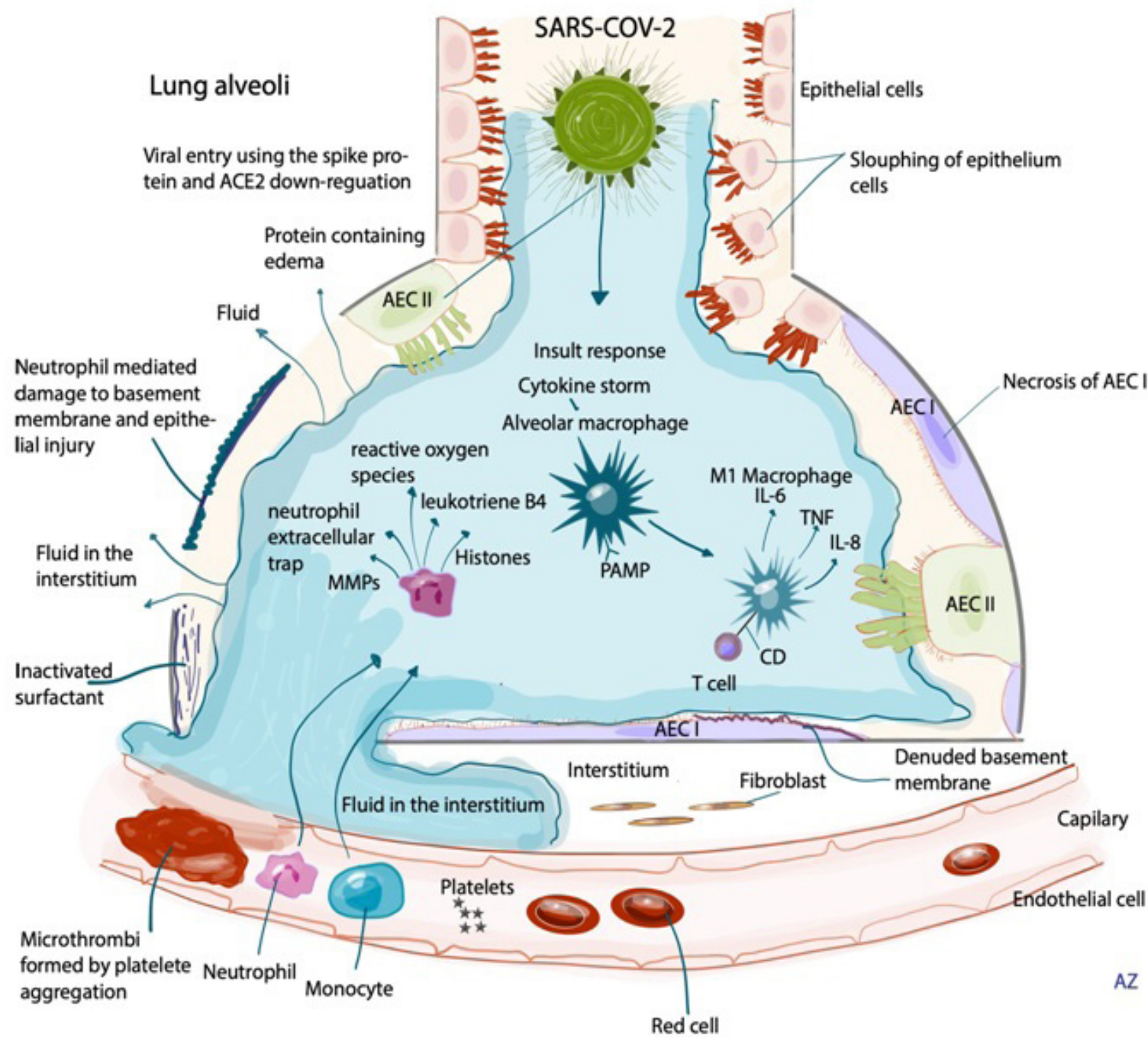

TNF - tumor necrosis factor; MMPs - matrix metalloproteinase; AECl - type I alveolar epithelial cell; AECII type II alveolar epithelial cell; PAMP - pathogen-associated molecular pattern;

Figure 1: Proposed Pathogenesis of SARS-Cov-2 Causing Acute Respiratory Distress. This is an image of an acute inflammatory response in the lung alveolus. The inflammatory response to SARS-CoV-2 leads to denuding of the basement membrane and disruption of epithelial barriers leading to accumulation of fluid in the alveoli. Alveolar macrophages are denoted above and release proinflammatory cytokines increasing neutrophil and monocyte infiltration. Alveolar macrophages release IL-6, TNF, IL-8 and other mediators contributing to an inflammatory cascade and cytokine storm. Neutrophils release reactive oxygen species (ROS), matrix metalloproteinases (MMPs), Leukotriene B4 (LTB4), neutrophil extracellular trap (NET) and other mediators. Tissue necrosis factor (TNF) increases an expression of tissue factor that contributes to platelet aggregation. Platelet aggregation leads to microthrombi and thrombi formation. Increased endothelial injury and increases coagulability and along with platelet aggregation lead to a hyper-thrombotic state AECI (type I alveolar epithelial cell) and AECII (type II alveolar epithelial cell). 
Citation: Zhyvotovska A, Yusupov D, Foronjy R, Nakeshbandi M, McFarlane SI, et al. (2020) Insights into Potential Mechanisms of Injury and Treatment Targets in COVID-19, SARS-Cov-2 Infection. Int J Clin Res Trials 5: 147. doi: https://doi.org/10.15344/2456-8007/2020/147

Page 3 of 9

SARS-CoV-2 infection results in reduced levels of peripheral CD4+ Tcells and CD8+ T cells though they remain hyperactivated $[14,16]$. CD8+ T cells are highly cytotoxic. Dysregulation of T cells leads to alveolar barrier destruction. Studies on SARS-CoV showed that the virus had an affinity for lymphocytes and numbers of both CD4+ Tcells and CD8+ T cells were reduced [14]. In a cohort of 191 patients, Zhou et al. found that COVID-19 non-survivors had a significantly lower baseline lymphocyte count than survivors [17]. A relative disruption in the number of $\mathrm{CD} 4+\mathrm{T}$ cells, often times the gate keepers of the immune response, leads to dysregulation of the entire immune system [7]. Elevated levels of biomarkers interleukin-6 (IL-6), tumor necrosis factor-alpha (TNF- $\alpha$ ), among others, are a sign of severe disease, and are associated with increased mortality [7]. CD8+ T cells damage the alveolar epithelial cells by secreting cytotoxic granules including granzymes that enter the cytoplasm of the target cell and trigger the caspase cascade, which eventually leads to apoptosis [18]. Apoptosis is also induced by cell-surface interaction between the TC and the infected cell by the Fas ligand on the infected cell binding to the Fas molecule that is expressed on the T cell [18]. Furthermore, damage to the alveolar type II cell in the lung has multiple consequences including inactivation of surfactant production, which worsens barrier disruption and alveolar edema and leads to accumulation of proteinrich fluid within the interstitium and alveoli [5,18]. Additionally, loss of surfactant has a detrimental effect on the elasticity of the lung tissue.

The immune dysregulation that occurs in some patients infected with SARS-CoV-2 has been termed, "Cytokine storm." This term, since the beginning of the pandemic, has been oft used but also poorly understood. Cytokine release syndrome (CRS), another name for this disorder, occurs in various conditions, including bacterial sepsis, in patients treated with chimeric antigen receptor (CAR) T cell therapy and hemophagocytic syndrome [19,20]. CRS causes increased

\section{Proposed Pathogenesis of Hemoglobin Dysfunction and T Cell Dysregulation}

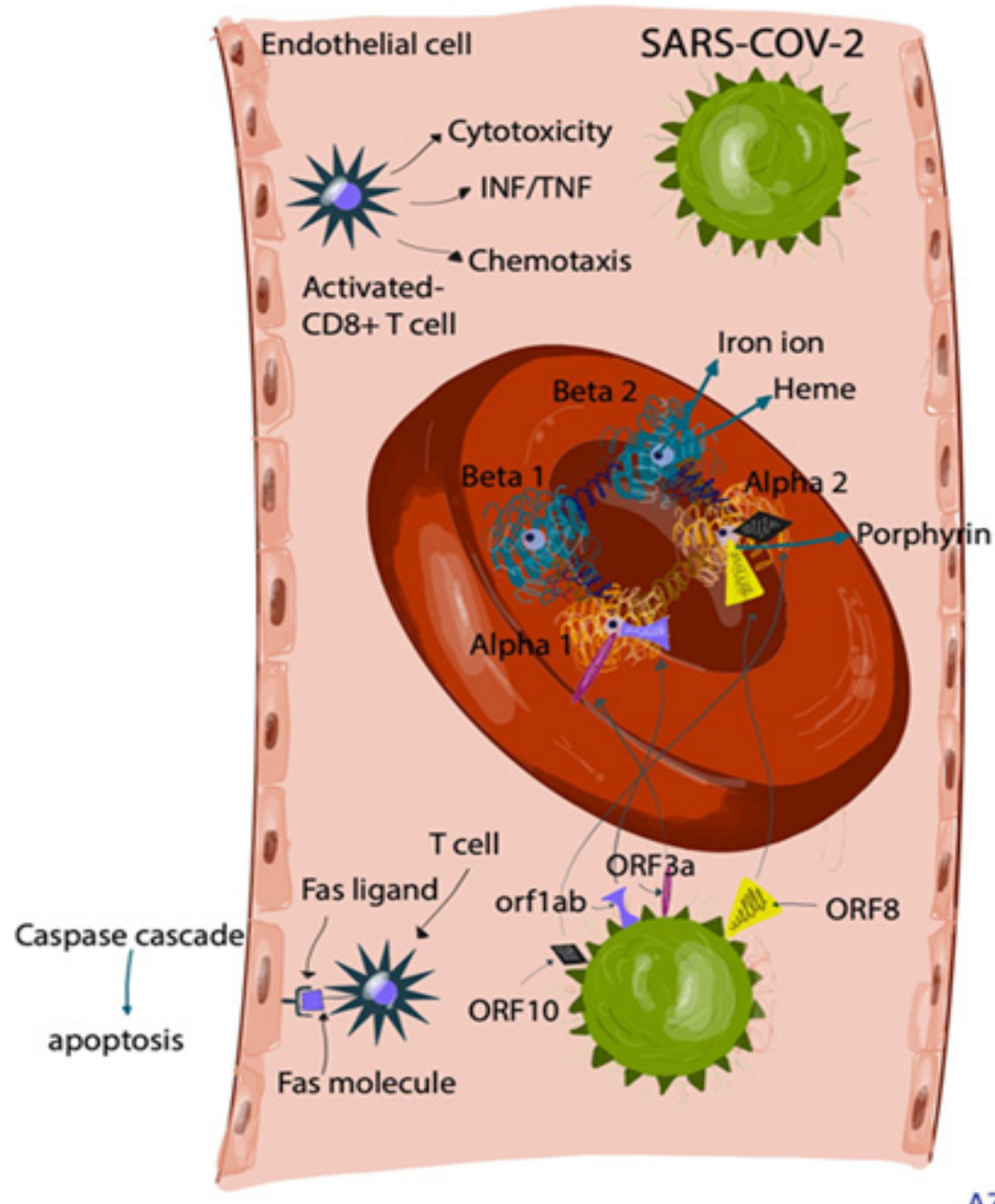

INF- interferon; TNF - tumor necrosis factor

Figure 2: Proposed pathogenesis of Hemoglobin Disruptions and T cell Dysregulation. Denoted in this image is the process of SARS-Cov-2 proteins: ORF8, ORF10, orf1ab, ORF3a interacting with heme component of the hemoglobin disrupting effective tissue oxygen delivery. Represented in this image are CD8 T cells that contribute to cytotoxicity, TNF and interferon (INF) release and chemotaxis. Cell-surface interaction between the $\mathrm{T}$ cells and the infected cell by the Fas ligand on the infected cell binding to the Fas molecule that is expressed on the $\mathrm{T}$ cell leads to apoptosis. 
Citation: Zhyvotovska A, Yusupov D, Foronjy R, Nakeshbandi M, McFarlane SI, et al. (2020) Insights into Potential Mechanisms of Injury and Treatment Targets in COVID-19, SARS-Cov-2 Infection. Int J Clin Res Trials 5: 147. doi: https://doi.org/10.15344/2456-8007/2020/147

Page 4 of 9

systemic inflammation, increased vascular permeability which contributes to the formation of pleural effusions and edema; CRS also leads to intravascular depletion via third space fluid loss and hypotension [20]. IL-6 is perhaps the most important cytokine in CRS. It has been found that in patients infected with SARS-CoV-2 elevated levels of IL-6 correlate with development of ARDS, adverse clinical outcomes and death [19-21].

Increased coagulation activity, marked by increased d-dimer concentrations, in some patients with COVID-19, further disrupts oxygenation. High levels of d-dimer have a reported association with increased mortality [17,22]. Inflammation and endothelial damage further increases procoagulant factors causing further endovascular damage and potentially eventual thrombosis and ischemia [8-12]. Laboratory test findings of 3 patients infected with SARS-CoV-2 at Tongji Hospital included leukocytosis, elevated prothrombin (PT) and partial prothrombin time (PTT), elevated fibrinogen and d-dimer levels, the presence of anticardiolipin $\operatorname{IgA}$ antibodies, anti-beta2-glycoprotein I IgA and IgG antibodies [12]. Most of these antibodies are part of antiphospholipid syndrome and can lead to thrombotic events in rheumatological diseases. They can also rise in response to various viral infections such as hepatitis $\mathrm{C}$ virus, human immunodeficiency virus, cytomegalovirus, varicella zoster, Epstein-Barr virus, adenovirus, and parvovirus B. In many instances, the presence of these antibodies was associated with thrombosis $[12,23,24]$. This is a potential mechanism of thrombosis in patients suffering from COVID-19 as well. IL-6, among other interleukins, is known to cause platelet hyperactivity, clumping and aggregation [25]. Furthermore, tumor necrosis factor (TNF) mediated expression of tissue factor promotes platelet aggregation and multifocal thrombi formation and interalveolar coagulation [25]. This occurs because TNF activates vascular monocytes and vascular endothelial cells to express tissue factor on their cell surfaces. This activates the extrinsic pathway of the coagulation system to induce microthrombus formation. Another pathway by which TNF contributes to microthrombus formation is by decreasing the endothelial expression of thrombomodulin and glycosaminoglycans that regulate the coagulation system $[23,25]$. All these dysregulations can lead to disseminated intravascular coagulation (DIC). TNF and other cytokines activate neutrophils to release various inflammatory mediators such as neutrophil extracellular trap, histones, leukotriene B4, MMP matrix metalloproteinase, MPO myeloperoxidase, reactive oxygen species and others which are capable of further damaging the endothelial cells [23].

Another potential mechanism of hypoxia was proposed by Liu et al. [26]. After performing a molecular analysis, it was discovered that COVID-19 possesses ORF8, ORF10, orf1ab, ORF3a and surface glycoproteins that interact with heme component of the hemoglobin. Each heme group contains iron ions that are attached to porphyrin. These iron ions participate in oxygen and carbon dioxide exchange in the body. ORF8 proteins have a capability to bind to the porphyrin in the heme. ORF10, orf1ab and ORF3a proteins can dissociate iron from the heme molecule of beta 1 hemoglobin chain (Figure 2). This way hemoglobin loses its ability to effectively deliver oxygen [26] and therefore some of the circulating hemoglobin becomes nonfunctional, contributing to further hypoxia. This is analogous to carbon monoxide poisoning. The dysfunctional hemoglobin causes the red blood cells to lose rheology and become deformed. This is potentially another contributing factor to hypoxia and thrombosis in the microcirculation. It is postulated that hydroxychloroquine inhibits the de-ironization of iron from heme [20], thereby preserving the oxygen carrying capacity of hemoglobin. Although the initial injury may occur in the lungs, the systemic inflammation and hypoxia have severe effects on other organs like the heart and kidneys. The evidence regarding this mechanism is rather limited at this time; both experimental and clinical trials are needed.

\section{Proposed Pathogenesis of SARS-CoV-2 and the Heart}

The exact mechanisms of cardiac injury in COVID-19 are not well established but the following outlines some of the likely causes. The cardiac effects are partially due to the reduction of ACE2, inflammatory responses, hypoxia, and disruptions in the coagulation pathway (Figure 3). ACE2 is widely expressed in cardiomyocytes, cardiac fibroblasts and coronary endothelial cells [2]. The increase of angiotensin II relative to angiotensin 1-7 due to COVID-19 has many deleterious effects in the heart. Angiotensin 1-7 exhibits antiproliferative, antiapoptotic, and mild vasodilating abilities and also has protective effects including those protective against heart failure, thrombosis, myocardial hypertrophy, fibrosis, arrhythmia, atherogenesis and also attenuates vascular dysfunction related to metabolic syndrome.2Other viral diseases like influenza and respiratory syncytial virus (RSV) have been associated with cardiac complications and poor outcomes [10,27]. There are several autopsy reports that showed acute myocardial infarction in patients infected with SARS-CoV-2. Zhou et al. reported that in a cohort of 191 patients infected with SARS-CoV-2, increased high-sensitivity cardiac troponin I was found in more than half of patients who died [17].

Shi et al. found that in a study of 416 patients, those with cardiac injury had a higher mortality than those who did not, $51.2 \%$ vs $4.5 \%$ [28]. It was found to be an independent predictor of mortality with a hazard ratio of 4.26 [28]. The potential causes are many-fold. Systemic inflammatory response can lead to increased inflammatory activity within coronary atherosclerotic plaques. This, in conjunction to endothelial dysfunction, increases the risk of plaque rupture [28]. Conversely, in studies in which patients, infected with SARSCoV-2, were found to have favorable troponin levels, they also more commonly had favorable outcomes $[28,29]$. The inflammatory response likely plays a large role in the myocarditis that develops in some patients infected with SARS-CoV-2. Autopsy reports have shown inflammatory infiltrates including an abundance of macrophages and some CD4+ T cells [7]. Though, as of this writing, there have not been reports of SARS-CoV-2 within myocardial tissue; however, viral genome was found in $35 \%$ of patients, in one study, who died from SARS-CoV [30].

Due to the difficulty of obtaining regular echocardiography's in patients with COVID-19, it is not possible to say with confidence whether there is a greater propensity of heart failure with preserved ejection fraction (HFpEF) or reduced ejection fraction (HFrEF). However, heart-failure symptoms are common in patients suffering from COVID-19. Zhou et al. described heart failure in $23 \%$ of their cohort, and in $52 \%$ of their non-survivors. In addition to the potential direct effects of COVID-19, the inflammatory response, the prothrombotic state that arises due to the disease, one must also take into the consideration the treatments currently being offered to patients infected with SARS-CoV-2, many of which increase the QTc interval. This includes but is not limited to the anti-malarial medications chloroquine and hydroxychloroquine, the antiviral agents, and the antibiotics such as azithromycin. 
Citation: Zhyvotovska A, Yusupov D, Foronjy R, Nakeshbandi M, McFarlane SI, et al. (2020) Insights into Potential Mechanisms of Injury and Treatment Targets in COVID-19, SARS-Cov-2 Infection. Int J Clin Res Trials 5: 147. doi: https://doi.org/10.15344/2456-8007/2020/147

Page 5 of 9

\section{Proposed Pathogenesis of SARS-CoV-2 and the Kidney}

Inflammation, vasoconstriction and the propensity for thrombosis following SARS-CoV-2 infection are systemic in nature and affect the kidneys in many ways. As more studies are published regarding COVID-19, the incidence of acute kidney injury (AKI) in patients infected with SARS-CoV-2 will be clearer. Co-expression of the ACE2 and TMPRSS genes has been found to be no less in the kidneys than that of the lungs and this suggests that kidney cells may be an important target for SARS-CoV-2 [3]. Current literature suggests that
SARS-CoV-2 displays a tropism towards the kidneys [31]. As of this writing, work by Yang et al., has shown that in a study of 52 critically ill adult patients, $23 \%$ suffered from AKI [32]. Zhou et al. found that AKI was found in $15 \%$ of their cohort overall, and in $50 \%$ of their non-survivors. There is relatively high co-expression of ACE2 and TMPRSS in podocytes and in the proximal straight tubules. Podocyte and proximal tubule destruction can explain why proteinuria and hematuria was seen in many patients with COVID-19 on presentation, $43.9 \%$ and $26.7 \%$, respectively, in a prospective cohort study of 701 patients by Cheng et al [33]. Previous studies on ACE-2 and renal cells

\section{Proposed Etiologies of Cardiac Pathologies of SARS-COV-2}

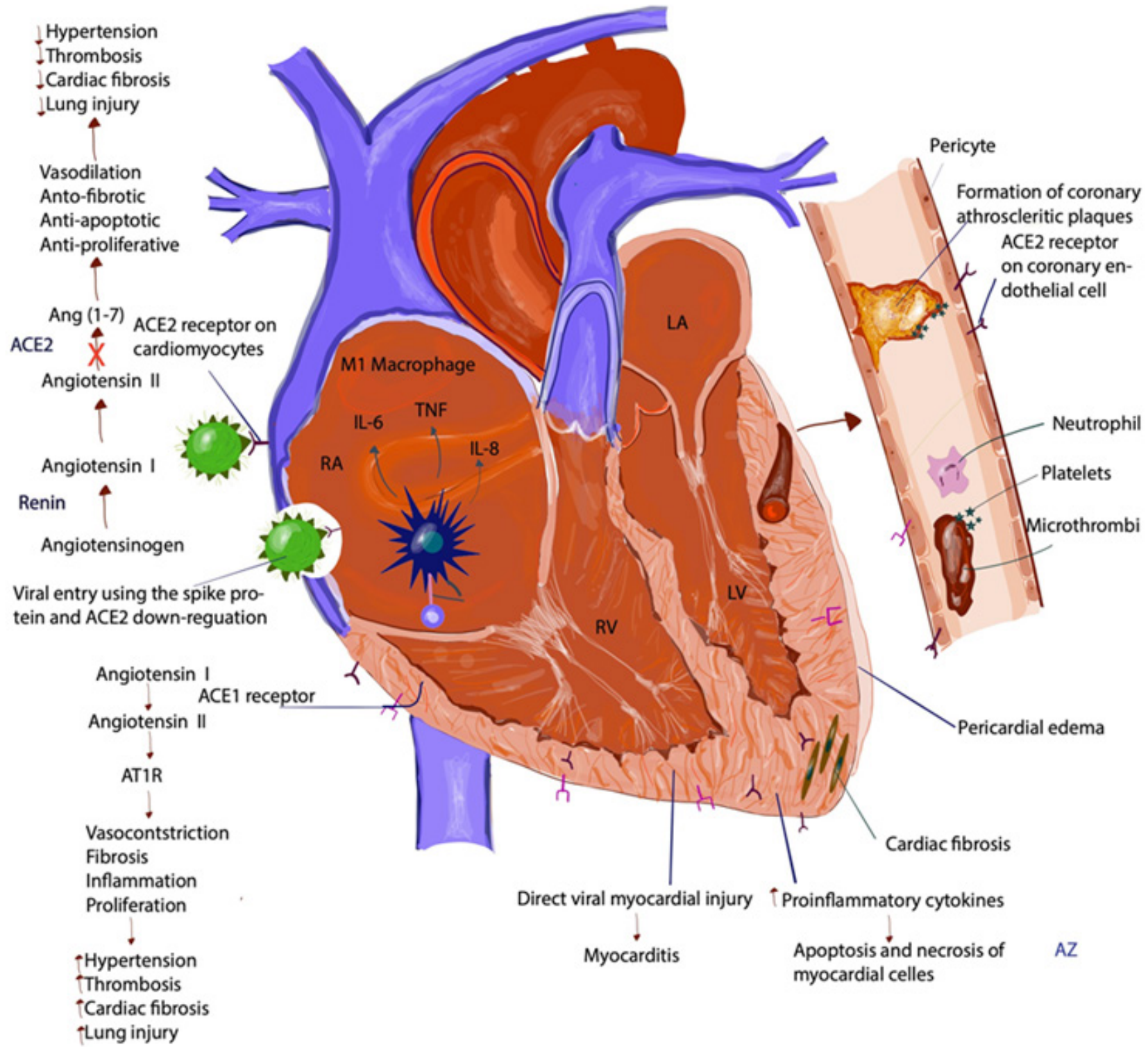

Figure 3: Proposed Etiologies of Cardiac Pathologies of SARS-CoV-2. This image describes the Renin-Angiotensin- Aldosterone (RAAS) pathway. Angiotensinogen is converted to Angiotensin I by renin, angiotensin I is converted to angiotensin 2 (Ang 2) by angiotensin converting enzyme 1 (ACE 1). ACE 2 is converted to angiotensin 1-7 (Ang 1-7) by angiotensin converting enzyme 2 (ACE 2). Since ACE 2 is downregulated by the virus, Ang 2 is not converted to Ang 1-7 as denoted by the ' $\mathrm{X}$ ' in the image. Ang 1-7 is anti-fibrotic, anti-proliferative, anti-apoptotic and vasodilatory; its downregulation leads to increased fibrosis, increased thrombosis, increased lung injury, increased blood pressure. It is postulated that direct myocardial injury by the virus can lead to myocarditis. Increase in pro-inflammatory cytokines leads to apoptosis and necrosis of myocardial cells. Increased inflammation leads to an accelerated formation of the atherosclerotic plaques and their rupture. Infiltration of the inflammatory cells in the pericardium leads to increased edema and pericarditis. 
Citation: Zhyvotovska A, Yusupov D, Foronjy R, Nakeshbandi M, McFarlane SI, et al. (2020) Insights into Potential Mechanisms of Injury and Treatment Targets in COVID-19, SARS-Cov-2 Infection. Int J Clin Res Trials 5: 147. doi: https://doi.org/10.15344/2456-8007/2020/147

in mice models has shown that inhibition of ACE2 leads to increased albuminuria and glomerular matrix expansion, increased mesangial matrix deposition, glomerular basement membrane thickening and glomerulosclerosis [34]. On the other hand, amplification of ACE2 has been shown to reduce the effect of nephropathy in mouse models[4]. Additionally, the inflammatory response described previously may play a profound effect on the kidneys (Figure 4).

Moreover, if renal tubular epithelium is injured, it may worsen cytokine overproduction [20]. The mechanism of this is not well understood; however, it has been observed that injury to tubular epithelium cells promotes the upregulation of IL-6 in human and animal studies [20]. Also of interest, is that podocytes are one of few cells to express IL-6 receptors on their surface thus they can directly respond to IL-6, and these receptors are upregulated during inflammatory processes [35]. We propose that this upregulation of the immune response in turn can cause further lung injury which may in turn lead to more profound hypoxic renal medullary injury.
Worsening cardiac function can also explain some of the AKI that occurs, as cardiomyopathy and myocarditis may ultimately lead to renal vein congestion and renal arterial hypoperfusion. These mechanisms, along with fluid imbalances, high airway pressures may lead to a renal compartment syndrome [20]. The disruption of the coagulation pathway and thrombus formation as described earlier likely also plays a role in the development of AKI in many patients with COVID-19. Additionally, as with all critically ill patients with a dysregulated immune response, the risk of superimposed bacterial infections, especially in patients with foley catheters, is high and warrants monitoring and consideration.

\section{Current Understanding of Therapeutic Targets and Treatment Options}

Figure 5 summarizes the proposed four pathophysiologic mechanisms of injury that culminates into organ damage following SARS-CoV-2 infection and what is currently in consideration as an

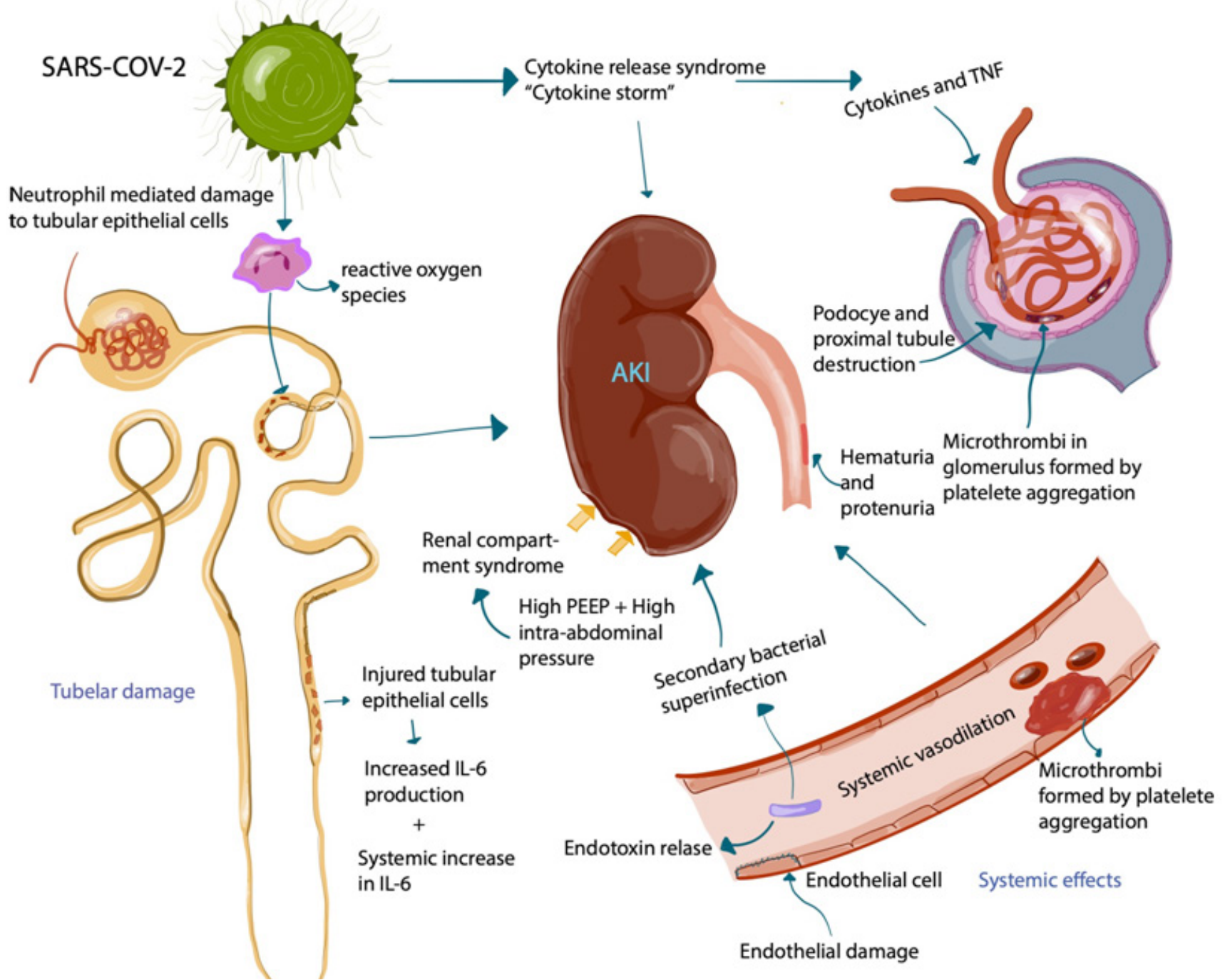

Figure 4: Proposed Pathogenesis of SARS-Cov-2 Causing Acute Kidney Injury (AKI). This is an image depicts the acute inflammatory response and systemic effects of SARS-CoV-2 on the kidneys. ACE2 receptors are found mostly on podocytes and tubular epithelial cells. Cellular internalization leads to glomerular and tubular damage. Innate and adapted immune response leads to systemic vasodilation and increased vascular permeability leading to further glomerular and tubular damage. Damage to renal tubular cells can worsen systemic inflammatory manifestations. The immune response also contributes to thrombus formation. Intragenic infections and superimposed bacterial infections are potential mediators of AKI. Intubated patients on high positive endexpiratory pressure (PEEP) settings may develop high intra-abdominal pressures potentially leading to renal compartment syndrome. 
Citation: Zhyvotovska A, Yusupov D, Foronjy R, Nakeshbandi M, McFarlane SI, et al. (2020) Insights into Potential Mechanisms of Injury and Treatment Targets in COVID-19, SARS-Cov-2 Infection. Int J Clin Res Trials 5: 147. doi: https://doi.org/10.15344/2456-8007/2020/147

Page 7 of 9

approach to therapy. It is not clear why $80 \%$ of the population will clear the virus at onset but others progress to downstream effects provoking strong inflammatory response and vasoconstriction that then increases the risk for thrombotic events and organ injury. Nonetheless, it appears that the inflammatory and vasoconstrictive responses are tightly linked and occur simultaneously. The inflammatory and vasoconstrictive responses, along with a hypercoagulable state induced by procoagulants produced as a result of the infection is a perfect setup for microcirculatory thrombosis and ultimately end organ damage. In the lungs this leads to acute respiratory distress syndrome (ARDS), with ventilation perfusion mismatches and explains why the ARDS in these patients does not respond as expected to high pressure mechanical ventilation support systems. To address COVID-19, there is opportunity to treat with agents that directly inhibit SARS-CoV-2 replication, such as antivirals (remdesivir)[36] macrolide antibiotics such as azithromycin and the anti-malarial hydroxychloroquine37 with caution [38], but randomized clinical trials to promote the widespread use of the agents as best practice are not yet available. The use of convalescent plasma [39] shows promise and is in clinical trials. It is important to note the pyrexia and diarrhea seen in these patients induces significant fluid deficits that must be replaced; however, care must be taken to not overhydrate these patients because of the risk of worsening ARDS. The ultimate goal in SARS-CoV-2 infection prevention, is vaccine development
[40] for which studies are underway. To address the inflammatory response, steroids [41] and the selective IL-6 blocker (Tocilizumab) [42] are in use but response is variable. The full spectrum of how the cytokine storm may be approached from lessons learned in influenza H1N1 is detailed by Liu et al. [43] and how immunosuppressive drugs affect COVID-19 response to therapy, including the use of JAK kinase inhibitors, is also thoroughly reviewed by Russel et al [44]. The use of non-specific anti-inflammatory or cell depleting agents is likely to attenuate host response, exacerbate viremia and should be discouraged. More recently the concept of using mesenchymal stem cells [45] for immunomodulatory and regenerative effects in COVID-19 infected patients is gaining consensus for clinical trials. To address the vasoconstriction, research into fusion inhibitor to block the virus from entering cells and administration of exogenous ACE2 to ameliorate the effects of angiotensin II seems prudent [15,45]. Preliminary observations of angiotensin II converting enzyme inhibitors (ACEI) angiotensin type 1 receptor blockers (ARB) in hospitalized patients have shown reduction in mortality [46] and therefore opens up another facet for clinical trials to be conducted. To address the thrombosis, all patients who progress to mechanical support and elevated d-dimers should be anticoagulated [47]. In our earlier encounter with these patients, this was not a consideration until more recently evidence in favor of anticoagulation has changed our management of these patients. The role of exchange transfusion

\section{Summary of Proposed Pathogenic Pathways and Treatment Targets}

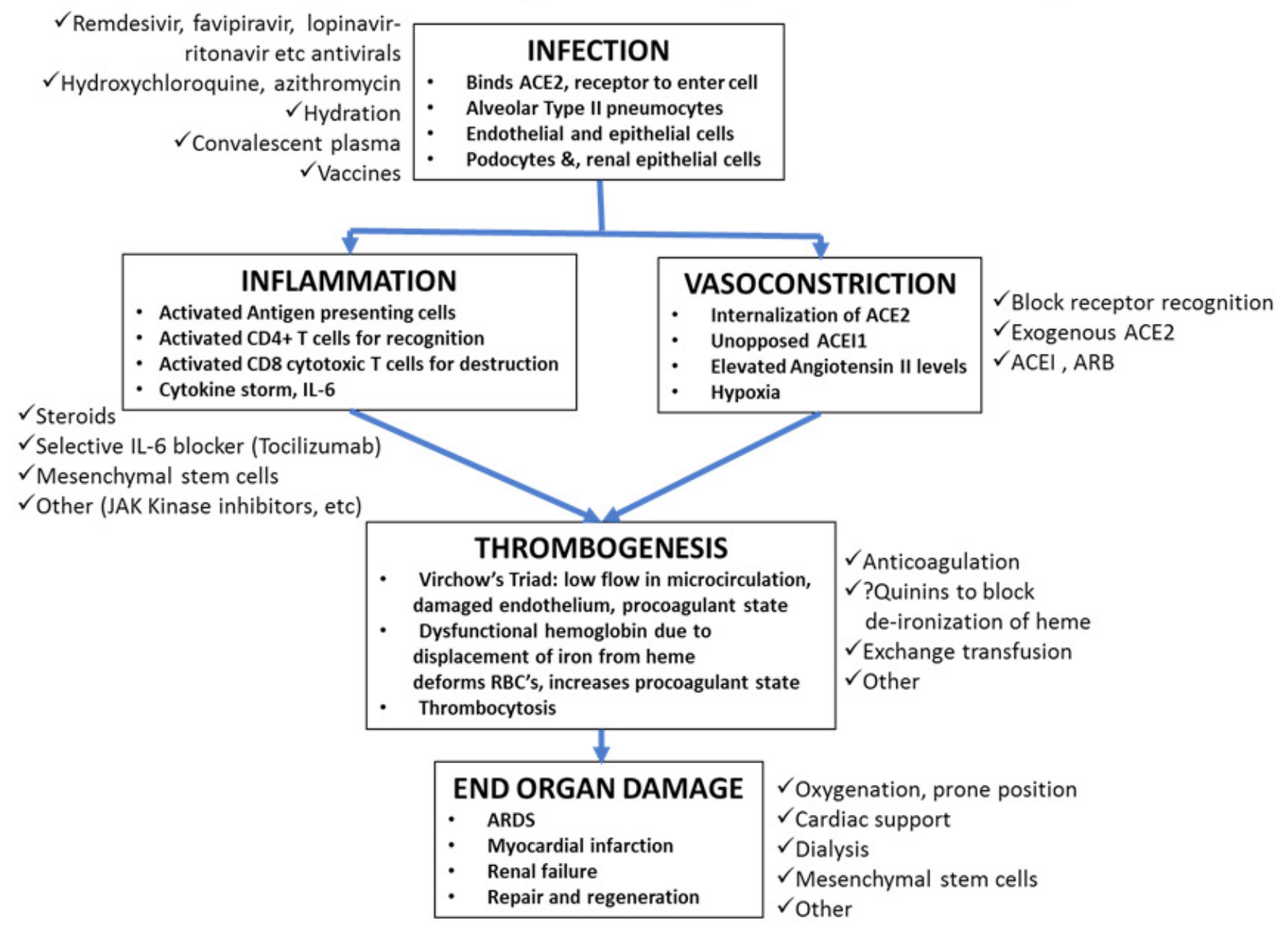

Figure 5: Illustration of the four pathogenic pathways that culminate into end organ damage. Treatment targets at each check point is illustrated outside the boxes. The illustrations underscores the need to address multiple check points in treating patients with COVID-19 infection. It also underscores the need for early intervention and more research into treatments that are not yet available but should be designed to address each of the check points. 
Citation: Zhyvotovska A, Yusupov D, Foronjy R, Nakeshbandi M, McFarlane SI, et al. (2020) Insights into Potential Mechanisms of Injury and Treatment Targets in COVID-19, SARS-Cov-2 Infection. Int J Clin Res Trials 5: 147. doi: https://doi.org/10.15344/2456-8007/2020/147

Page 8 of 9

to replace dysfunctional hemoglobin caused by de-ironization of heme in COVID-19 infection is yet to be determined and whether hydroxychloroquine helps stabilize iron in hemoglobin [48] thereby contributing to improvement in oxygenation is unclear. Finally, better understanding of the pathogenesis along with early interventions are critical in avoiding end organ damage. End organ damage requires support. In ARDS, lung protective ventilation (avoiding high positive end expiratory pressures) with high flow oxygen delivery, as well as physical therapy methods including being in the prone position [49] are the main stay of therapy, while in AKI, dialysis may be required. The use of cardiac support devices is limited in managing SARSCoV-2 infected patients, nonetheless as we gain better control of each check point and the ability to confirm conversion to negative tests, opportunities will avail for studies in cardiac support that may pave a way for lung transplantation or combined heart and lung transplantation. The concept of using mesenchymal stem cells [45] for immunomodulatory and regenerative effects in SARS-CoV-2 infected patients for lung repair following ARDS is gaining consensus for clinical trials.

It is important to emphasize that none of the therapeutics for SARSCoV-2 infection described here have been tested in randomized clinical trials and are at best investigational or used compassionately. It is also important to note that our attempt is to outline potential mechanisms of organ injury in SARS-CoV-2 and where treatments may be targeted and not an in-depth review of available treatments for COVID-19.

\section{Conclusion}

Despite the short experience of COVID-19 pandemic and acknowledging that much of the pathogenesis is yet to be uncovered, significant strides have been made in our current understanding of the pathogenesis of injury that has paved the way for us to illustrate the potential therapeutic targets. Each therapeutic target has an existing treatment options, but none has been approved for widespread use. Therapies to slow viral replication and certainly vaccine development for prevention are critical, nonetheless, it appears that a multi-faceted approach addressing some or all the proposed therapeutic targets is necessary to prevent or slow end organ injury. At this point, it is prudent to start treating a patient with COVID-19 based on or current understanding of pathogenesis, until future research unveils more insights into the pathogenesis and randomized clinical trials point us in a more structured direction for best practice.

\section{Competing Interests}

The authors declare that they have no competing interests.

\section{Acknowledgement}

This work is supported, in part, by the efforts of Dr. Moro O. Salifu MD, MPH, MBA, MACP, Professor and Chairman of Medicine through NIMHD Grant number S21MD012474.

\section{References}

1. Hoffmann $\mathrm{M}$, Kleine-Weber $\mathrm{H}$, Schroeder $\mathrm{S}$, Krüger $\mathrm{N}$, Herrler $\mathrm{T}$, et al. (2020) SARS-CoV-2 Cell Entry Depends on ACE2 and TMPRSS2 and Is Blocked by a Clinically Proven Protease Inhibitor. Cell 181: 271-280.

2. Guo J, Huang Z, Lin L, Lv J (2020) Coronavirus Disease 2019 (COVID-19) and Cardiovascular Disease: A Viewpoint on the Potential Influence of Angiotensin-Converting Enzyme Inhibitors/Angiotensin Receptor Blockers on Onset and Severity of Severe Acute Respiratory Syndrome Coronavirus 2 Infection. J Am Heart Assoc 9: e016219.
3. Pan XW, Xu D, Zhang $H$, Zhou W, Wang LH, et al. (2020) Identification of a potential mechanism of acute kidney injury during the COVID-19 outbreak: a study based on single-cell transcriptome analysis. Intensive Care Med.

4. Soler MJ, Wysocki J, Batlle D (2013) ACE2 alterations in kidney disease. Nephrol Dial Transplant 28: 2687-2697

5. Verdecchia P, Cavallini C, Spanevello A, Angeli F (2020) The pivotal link between ACE2 deficiency and SARS-CoV-2 infection. Eur J Intern Med.

6. Gurwitz D (2020) Angiotensin receptor blockers as tentative SARS-CoV-2 therapeutics. Drug Dev Res.

7. Akhmerov A, Marban E (2020) COVID-19 and the Heart. Circ Res 126: $1443-$ 1455.

8. Milbrandt EB, Reade MC, Lee MJ, Shook SL, Angus DC, et al. (2009) Prevalence and significance of coagulation abnormalities in communityacquired pneumonia. Mol Med 15: 438-445.

9. Smeeth L, Thomas SL, Hall AJ, Hubbard R, Farrington P, et al. (2004) Risk of myocardial infarction and stroke after acute infection or vaccination. N Engl J Med 351: 2611-2618.

10. Corrales-Medina VF, Musher DM, Shachkina S, Chirinos JA (2013) Acute pneumonia and the cardiovascular system. Lancet 381: 496-505.

11. Davidson JA, Warren-Gash C (2019) Cardiovascular complications of acute respiratory infections: current research and future directions. Expert Rev Anti Infect Ther 17: 939-942.

12. Zhang $Y$, Xiao $M$, Zhang $S$, Xia P, Cao W, et al. (2020) Coagulopathy and Antiphospholipid Antibodies in Patients with Covid-19. N Engl J Med 382: e38.

13. Gattinoni L, Coppola S, Cressoni M, Busana M, Rossi S, et al. (2020) Covid-19 Does Not Lead to a "Typical" Acute Respiratory Distress Syndrome. Am J Respir Crit Care Med 201: 299-1300.

14. Liu Y, Yang Y, Zhang C, Huang F, Wang F, et al. (2020) Clinical and biochemical indexes from 2019-nCoV infected patients linked to viral loads and lung injury. Sci China Life Sci 63: 364-374.

15. Zou Z, Yan Y, Shu Y, Gao R, Sun Y, et al. (2014) Angiotensin-converting enzyme 2 protects from lethal avian influenza A H5N1 infections. Nat Commun 5: 3594.

16. Xu Z, Shi L, Wang Y, Zhang J, Huang L, et al. (2020) Pathological findings of COVID-19 associated with acute respiratory distress syndrome. Lancet Respir Med 8: 420-422.

17. Zhou F, Yu T, Du R, Fan G, Liu Y, et al. (2020) Clinical course and risk factors for mortality of adult inpatients with COVID-19 in Wuhan, China: a retrospective cohort study. Lancet 395: 1054-1062.

18. Bhatia M, Moochhala S (2004) Role of inflammatory mediators in the pathophysiology of acute respiratory distress syndrome. J Pathol 202: 145156.

19. Moore JB, June CH (2020) Cytokine release syndrome in severe COVID-19. Science 368: 473-474

20. Ronco C, Reis T (2020) Kidney involvement in COVID-19 and rationale for extracorporeal therapies. Nat Rev Nephrol 16: 308-310.

21. Wu C, Chen X, Cai Y, Xia J, Zhou X, et al. (2020) Risk Factors Associated With Acute Respiratory Distress Syndrome and Death in Patients With Coronavirus Disease 2019 Pneumonia in Wuhan, China. JAMA Intern Med.

22. Zhang L, Yan X, Fan Q, Liu H, Liu X, et al. (2020) D-dimer levels on admission to predict in-hospital mortality in patients with Covid-19. J Thromb Haemost.

23. Uthman IW, Gharavi AE (2002) Viral infections and antiphospholipid antibodies. Semin Arthritis Rheum 31: 256-263.

24. Abdel-Wahab N, Talathi S, Lopez-Olivo MA, Suarez-Almazor ME (2018) Risk of developing antiphospholipid antibodies following viral infection: a systematic review and meta-analysis. Lupus 27: 572-583.

25. Page MJ, Bester J, Pretorius E (2018) The inflammatory effects of TNF-alpha and complement component 3 on coagulation. Sci Rep 8: 1812.

26. Liu W, Li H (2020) COVID-19: Attacks the 1-Beta Chain of Hemoglobin and Captures the Porphyrin to Inhibit Human Heme Metabolism. Chem Rxiv.

27. Blackburn R, Zhao H, Pebody R, Hayward A, Warren-Gash C, et al. (2018) Laboratory-Confirmed Respiratory Infections as Predictors of Hospital Admission for Myocardial Infarction and Stroke: Time-Series Analysis of English Data for 2004-2015. Clin Infect Dis 67: 8-17. 
Citation: Zhyvotovska A, Yusupov D, Foronjy R, Nakeshbandi M, McFarlane SI, et al. (2020) Insights into Potential Mechanisms of Injury and Treatment Targets in COVID-19, SARS-Cov-2 Infection. Int J Clin Res Trials 5: 147. doi: https://doi.org/10.15344/2456-8007/2020/147

28. Shi $\mathrm{S}$, Qin $\mathrm{M}$, Shen $\mathrm{B}$, Cai $Y$, Liu T, et al. (2020) Association of Cardiac Injury With Mortality in Hospitalized Patients With COVID-19 in Wuhan, China. JAMA Cardiol.

29. Guo T, Fan Y, Chen M, Wu X, Zhang L, et al. (2020) Cardiovascular Implications of Fatal Outcomes of Patients With Coronavirus Disease 2019 (COVID-19). JAMA Cardiol.

30. Oudit GY, Kassiri Z, Jiang C, Liu PP, Poutanen SM, et al. (2009) SARScoronavirus modulation of myocardial ACE2 expression and inflammation in patients with SARS. Eur J Clin Invest 39: 618-625.

31. Puelles VG, Lütgehetmann $M$, Lindenmeyer MT, Sperhake JP, Wong MN et al. (2020) Multiorgan and Renal Tropism of SARS-CoV-2. N Engl J Med.

32. Yang $X, Y u Y, X u J$, Shu $H, X i a ~ J$, et al. (2020) Clinical course and outcomes of critically ill patients with SARS-CoV-2 pneumonia in Wuhan, China: a singlecentered, retrospective, observational study. Lancet Respir Med 8: 475-481.

33. Cheng Y, Luo R, Wang K, Zhang M, Wang Z, et al. (2020) Kidney disease is associated with in-hospital death of patients with COVID-19. Kidney Int 97: 829-838.

34. Oudit GY, Herzenberg AM, Kassiri Z, Wong D, Reich $H_{\text {, et al. (2006) Loss }}$ of angiotensin-converting enzyme-2 leads to the late development of angiotensin II-dependent glomerulosclerosis. Am J Pathol 168: 1808-1820.

35. Su H, Lei CT, Zhang C (2017) Interleukin-6 Signaling Pathway and Its Role in Kidney Disease: An Update. Front Immunol 8: 405.

36. Grein J, Ohmagari N, Shin D, Diaz G, Asperges E, et al. (2020) Compassionate Use of Remdesivir for Patients with Severe Covid-19. N Engl J Med.

37. Gautret P, Lagier JC, Parola P, Hoang VT, Meddeb L, et al. (2020) Hydroxychloroquine and azithromycin as a treatment of COVID-19: results of an open-label non-randomized clinical trial. Int J Antimicrob Agents 2020:105949.

38. Sapp JL, Alqarawi W, MacIntyre CJ, Tadros R, Steinberg C, et al. (2020) Guidance On Minimizing Risk of Drug-Induced Ventricular Arrhythmia During Treatment of COVID-19: A Statement from the Canadian Heart Rhythm Society. Can J Cardiol.

39. Bloch EM, Shoham S, Casadevall A, Sachais BS, Shaz B, et al. (2020) Deployment of convalescent plasma for the prevention and treatment of COVID-19. J Clin Invest.

40. Ahn M, Park EJ, Woo JM (2017) Current status and future prospects of research and development operations in traditional and complementary and alternative medicine manufacturing small- and medium-sized enterprises: a 2014 company-based survey. Integr Med Res 6: 409-417.

41. Poston JT, Patel BK, Davis AM (2020) Management of Critically Ill Adults With COVID-19. JAMA

42. Luo $P$, Liu $Y$, Qiu L, Liu $X$, Liu $D$, et al. (2020) Tocilizumab treatment in COVID-19: A single center experience. J Med Virol.

43. Liu Q, Zhou YH, Yang ZQ (2016) The cytokine storm of severe influenza and development of immunomodulatory therapy. Cell Mol Immunol 13: 3-10.

44. Russell B, Moss C, George G, Santaolalla A, Cope A, et al. (2020) Associations between immune-suppressive and stimulating drugs and novel COVID-19-a systematic review of current evidence. Ecancermedicalscience 14: 1022.

45. Leng Z, Zhu R, Hou W, Feng Y, Yang Y, et al. (2020) Transplantation of ACE2(-) Mesenchymal Stem Cells Improves the Outcome of Patients with COVID-19 Pneumonia. Aging Dis 11: 216-228.

46. Zhang P, Zhu L, Cai J, Lei F, Qin JJ, et al. (2020) Association of Inpatient Use of Angiotensin Converting Enzyme Inhibitors and Angiotensin II Receptor Blockers with Mortality Among Patients With Hypertension Hospitalized With COVID-19. Circ Res 126: 1671-1681.

47. Barrett CD, Moore HB, Yaffe MB, Moore EE (2020) ISTH interim guidance on recognition and management of coagulopathy in COVID-19: A Comment. J Thromb Haemost.

48. Kiekens C, Boldrini P, Andreoli A, Avesani R, Gamna F, et al. (2020) Rehabilitation and respiratory management in the acute and early postacute phase. "Instant paper from the field" on rehabilitation answers to the Covid-19 emergency. Eur J Phys Rehabil Med.

49. Thompson BT, Chambers RC, Liu KD (2017) Acute Respiratory Distress Syndrome. N Engl J Med 377: 1904-1905. 